\title{
Intermittent negative pressure applied to the lower limb increases foot macrocirculatory and microcirculatory blood flow pulsatility in people with spinal cord injury
}

\author{
Øyvind Heiberg Sundby ${ }^{1,2,3} \cdot$ Lars $_{\text {Øivind Høiseth }}{ }^{1,4} \cdot$ Ingebjørg Irgens $^{2,5} \cdot$ lacob Mathiesen $^{3}$ • Eivind Lundgaard ${ }^{5}$. \\ Hanne Haugland $^{6} \cdot$ Harald Weedon-Fekjær $^{7} \cdot$ Jon O. Sundhagen ${ }^{8} \cdot$ Gunnar Sanbæk $^{2,9} \cdot$ Jonny Hisdal ${ }^{1}$
}

Received: 20 October 2017 / Revised: 2 December 2017 / Accepted: 4 December 2017 / Published online: 28 December 2017

(c) International Spinal Cord Society 2018

\begin{abstract}
Study design Experimental prestudy and poststudy.

Objectives Examine the acute effects of intermittent negative pressure (INP) applied to the lower limb on foot circulation in people with spinal cord injuries (SCIs).

Setting Vascular laboratory, Oslo University Hospital.

Methods Twenty-four people with SCI (median age 59 years, range 29-74) were exposed to lower leg INP (-40 mm Hg) using an air-tight pressure chamber connected to an INP generator. The contralateral leg was placed outside the pressure chamber. We continuously measured arterial blood flow velocity (ultrasound Doppler), skin blood flow (laser Doppler), skin temperature of the dorsum of the foot, heart rate (ECG) and systemic blood pressure (Finometer) during 5-min baseline (atmospheric pressure), followed by 10-min INP (alternating $10 \mathrm{~s}-40 \mathrm{~mm} \mathrm{Hg}$ and $7 \mathrm{~s}$ atmospheric pressure), and 5-min post-INP (atmospheric pressure). Skin blood flow was measured on the foot placed outside the pressure chamber. A mixed effects regression model was applied to estimate the effect of INP on blood flow. To quantify flow fluctuations, we calculated cumulative up-and-down changes in arterial blood flow velocity per minute.

Results Flow fluctuations increased during INP compared to baseline [32.3 cm/s/min (95\% CI 26.9 to 37.7 ) vs. $15.2 \mathrm{~cm} / \mathrm{s} /$ $\min (95 \%$ CI 9.8 to 20.6), $P<0.001]$. Peak blood flow velocity and skin blood flow was reached $2-3 \mathrm{~s}$ after the onset of negative pressure and increased $33 \%$ (95\% CI 16 to $46, P<0.001)$ and $11 \%(95 \% \mathrm{CI}-4.1$ to $60, P=0.14$ ) above baseline, respectively.
\end{abstract}

Conclusions INP induced increased foot arterial blood flow fluctuations compared to baseline.

Sponsorships The Norwegian Research Council provided funding to Otivio (grant: 241589).

\section{Introduction}

People with chronic spinal cord injuries (SCIs) experience delayed ulcer healing rates below the level of spinal

Øyvind Heiberg Sundby

oyvindheibergsundby@gmail.com

1 Section of Vascular Investigations, Department of Vascular Surgery, Oslo University Hospital, Oslo, Norway

2 Faculty of Medicine, Institute of Clinical Medicine, University of Oslo, Oslo, Norway

3 Otivio AS, Gaustadalléen 21, 0349 Oslo, Norway

4 Department of Anesthesiology, Oslo University Hospital, Oslo, Norway denervation compared to their able-bodied peers [1]. Poor ulcer healing rates among SCI people cannot be explained by loss of sensation and immobility alone, since skin ulcers frequently show delayed healing after pressure is removed
Department of Research, Sunnaas Rehabilitation Hospital, Oslo, Norway

6 Department of Spinal Cord Injury, Sunnaas Rehabilitation Hospital, Oslo, Norway

7 Oslo Center for Biostatistics and Epidemiology, Research Support Services, Oslo University Hospital, Oslo, Norway

8 Department of Vascular Surgery, Oslo University Hospital, Oslo, Norway

9 Department of Radiology, Oslo University Hospital, Oslo, Norway 
or further deterioration and ulcer development following minor trauma [2]. One important factor thought to contribute to poor healing rates in people with chronic SCI is ischemia, due to decreased blood supply and decreased blood flow of denervated cutaneous and muscle tissues [3].

Recent studies in healthy subjects [4] and in patients with peripheral arterial disease [5, 6] showed that applying intermittent negative pressure (INP; $-40 \mathrm{~mm} \mathrm{Hg}$ ) to the lower leg and foot increased foot macrocirculation and microcirculation. The increased foot circulation was due to INP-induced increases in blood flow pulsatility (variability in blood flow velocity and skin blood flow) [4, 5]. Foot circulation increased with only minor changes in central hemodynamics [4, 5].

The theoretical foundation for the use of INP is that it improves blood flow to the small blood vessels of the skin by inducing rapid changes in perfusion pressure at the onset of negative pressure [7, 8]. This increases the delivery of oxygen and nutrients to the cells, thereby facilitating wound healing [6]. Several clinical studies suggest that INP improves wound healing of chronic ulcers [6, 7] and increases peripheral circulation [9-11].

Loss of supraspinal regulation in people with SCI results in deficient control of the vascular bed below the spinal cord lesion [12, 13] and enhanced vasoconstrictor response during leg dependency [14]. Together, these factors may potentially cause different cardiovascular responses during INP in people with SCI compared to their able-bodied peers. Although previous studies show increased acute and longterm circulatory effects in the treated limb during application of INP [4, 5, 7], the effects of INP on foot circulation and central hemodynamics in people with SCI are unknown. The aim of the present study was therefore to investigate the acute hemodynamic effects of lower limb INP (-40 mm Hg) in people with SCI. We hypothesized that application of INP would increase arterial and skin blood flow pulsatility in the foot compared to atmospheric pressure (baseline), with only minor changes in central hemodynamics.

\section{Methods}

\section{Recruitment}

Eligible participants with chronic SCI were recruited from the Norwegian Spinal Cord Injury Association and the weekly exercise training groups for people with SCI at Oslo University Hospital, Aker between November 2015 and March 2016.

\section{Participants}

Inclusion criteria were: (i) complete or incomplete SCI; and (ii) $>6$ months since SCI. Exclusion criteria were: (i) chronic respiratory insufficiency; (ii) eczema and psoriasis; (iii) uncontrolled hypertension; and (iv) recent (<12 months) vascular, abdominal, cardiothoracic, or lower limb vascular surgery. Neurological levels were classified as complete or incomplete according to the American Spinal Injury Association classification system [15]. All participants were asked to refrain from eating $2 \mathrm{~h}$ before the experiment and from consuming alcohol, tobacco, or caffeine on the day of the experiment. None of the participants had signs of active lower extremity infections or ulcers.

\section{Vascular measurements before the experiments}

Participants were asked to empty their urinary bladder prior to having their vascular measurements taken, to minimize sympathetic activity from bladder distension on vascular tone [16]. After participants rested for $5 \mathrm{~min}$ in a supine position, we performed an initial vascular assessment to measure limb hemodynamics, using ankle-brachial pressure index (ABPI) with a continuous-wave hand-held $8 \mathrm{MHz}$ Doppler blood velocity detector (MacroLab; STR Teknikk, Aalesund, Norway) [17]. The systolic pressures were measured in the following order: right brachial, dorsalis pedis, and posterior tibial arteries; left dorsalis pedis and posterior tibial arteries; and the left brachial artery. Thereafter, ankle pulse-volume recording (PVR) was measured in both ankles with pressure cuffs connected to an air plethysmograph (Stranden MacroLab, STR Teknikk, Aalesund, Norway). PVR is a non-invasive test to evaluate total inflow in the examined limb. The pressure cuffs compress the veins, but not the arteries, and sense pulsatile arterial inflow with each cardiac cycle without venous interference [18].

In accordance with previous investigations on patients with peripheral arterial disease [5], showing that lower extremities with low systolic pressure are the most vulnerable to developing arterial leg ulcers [19], we chose the lower extremity with the lowest systolic ankle pressure as the test leg during the experiments (limb exposed to INP).

\section{Signal acquisition and analysis during the experiments}

Figure 1 shows the probes attached to the foot to assess foot circulation. During the experiment, arterial blood flow velocity was continuously measured in the dorsalis pedis artery with a $10 \mathrm{MHz}$ pulsed Doppler probe (SD-50; GE Vingmed Ultrasound, Horten, Norway). If it was not possible to obtain an adequate blood flow signal in the dorsal pedis artery, the posterior tibial artery was used. The ultrasound beam transducer was positioned centrally at either the dorsum of the foot or posterior to the medial malleolus of the ankle, depending on where we obtained the 

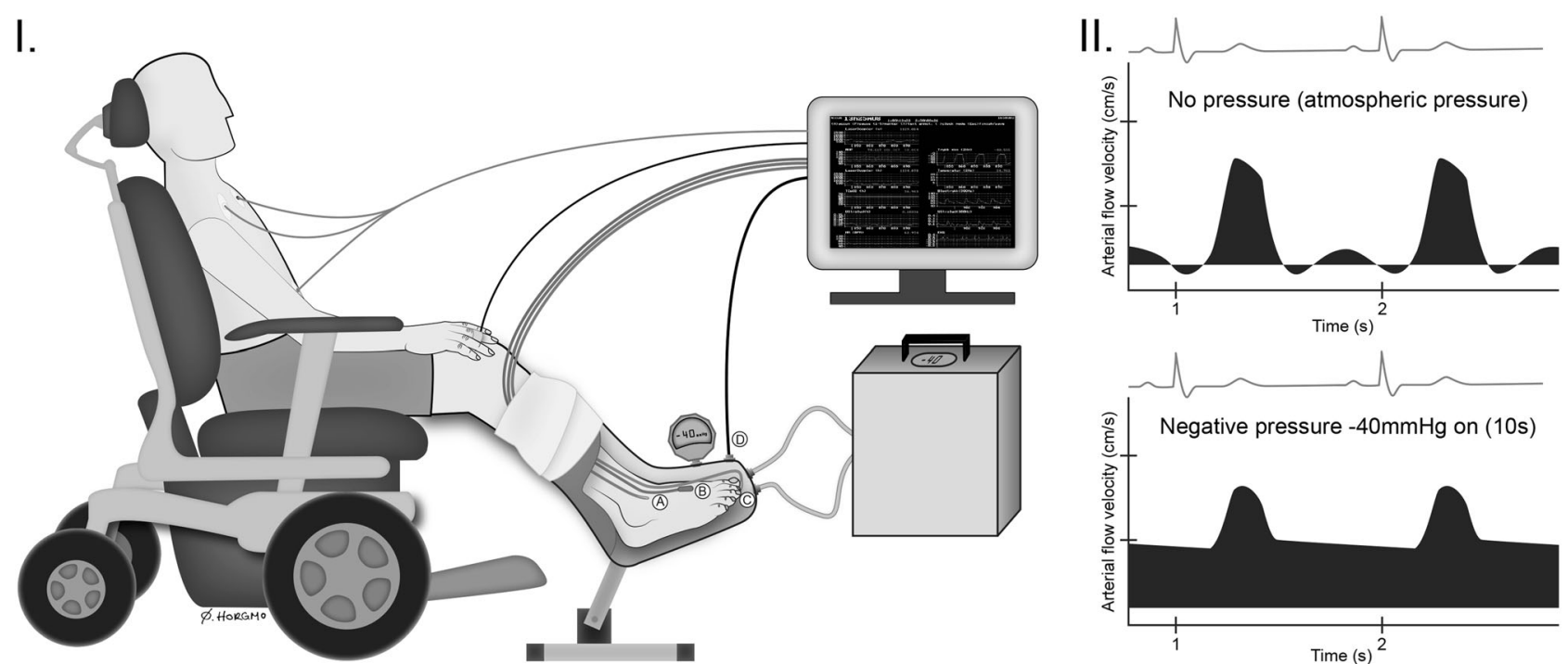

Fig. 1 I: Illustration of the experimental setup with probes attached to the foot. The participant's lower limb (test leg) was placed in the custom-made subatmospheric pressure chamber interfaced with the pressure control system. The subatmospheric pressure chamber was sealed around the participant's leg below the knee. The contralateral leg was placed outside the air-tight subatmospheric pressure chamber. The participant was connected to a three-lead ECG and a Finometer to continuously measure blood pressure. A: Skin temperature probe; B: Ultrasound Doppler probe; C: Laser Doppler flux probe; D: Pressure

highest blood flow velocity signal. Thereafter, the transducer was attached to the skin by surgical tape (Micropore Surgical Tape, 3M, MN, US).

After preparing the pulp skin of the first toes with an alcohol swab, laser Doppler fluxmetry (LDF) probes (404-1 and Periflux 4000; Perimed AB, Järfälla, Sweden) were attached bilaterally to the skin of the pulps of the first toes (Fig. 1). LDF gives a semiquantitative measurement of cutaneous peripheral microcirculation expressed in arbitrary units (AU) [20].

Skin temperature was measured with temperature probes (AD590 temperature transducer, STR Teknikk, Aalesund, Norway) attached to the dorsum of the foot using surgical tape.

Finger arterial pressure was measured continuously via a finger cuff from the third finger of the right hand, using a photoplethysmographic volume-clamp method (Finometer; FMS Finapres Medical Systems BV, Amsterdam, The Netherlands). Care was taken to adjust the arm so that the finger was at the level of the heart. Finometer pressures were calibrated with an automated sphygmomanometer (Solar 8000i, GE-Marquette Medical Systems, Inc., Milwaukee, USA) at the beginning of each experiment. Instantaneous heart rate (HR) was obtained from the duration of each R-R interval of the three-lead electrocardiogram (ECG) signal. transducer from subatmospheric pressure interfaced with the computer. An additional external calibrated pressure gauge was connected to the subatmospheric pressure in order to calibrate the data program (REGIST 3) before each experiment. II: Example of typical arterial blood flow velocity response in the dorsal pedis artery/tibial posterior artery during application of INP, with the diastolic velocities seemingly contributing the most. Illustration: Øystein H. Horgmo, University of Oslo

After attaching the probes, the participants' feet were covered with loose, non-elastic wool socks to keep them warm and under approximately constant temperature during the course of the study. Also, a light blanket covered the participants during the experiments to avoid cooling.

Analog signals for all measurements were continuously sampled at $300 \mathrm{~Hz}$ and averaged beat-by-beat gated by the R-waves of the three-lead ECG using custom-made software (REGIST 3, Morten Eriksen, University of Oslo, Oslo, Norway). Thereafter, all physiological data were imported to a personal computer and Microsoft Excel (Microsoft Office 2010 for Windows; Microsoft, Redmond, Washington, USA) for further analysis. Participants were instructed to avoid moving and talking throughout the sampling period. All measurements were performed by the same researcher.

\section{INP application}

The experimental set-up is illustrated in Fig. 1. It was recently described in detail together with the experimental protocol elsewhere [5]. Briefly, after attaching the ECG, blood pressure cuff, and probes to the foot, the participants were comfortably clothed and seated in their wheelchair for $20 \mathrm{~min}$ before the experiment started. The test leg was placed in a rigid molded polyethylene custom-made subatmospheric pressure chamber connected to a pressure 
control system (FlowOx, Otivio AS, Oslo, Norway). The contralateral leg was placed outside the subatmospheric pressure chamber. Internal padding in the subatmospheric pressure chamber allowed for insertion of the leg with probes attached, preventing pressure points on the skin of the leg and foot. Only the posterior part of the lower leg was in contact with the internal padding. To allow for application of negative pressure, the subatmospheric pressure chamber was sealed just below the knee with a thermoplastic elastomer (TPS-SEBS) (Fig. 1). During the experiments, the participants were asked to sit with an approximate angle of $130^{\circ}$ in their knee and hip joints.

\section{Experimental design}

After 5-min baseline registrations with no pressure manipulation (atmospheric pressure), INP with $-40 \mathrm{~mm} \mathrm{Hg}$ negative pressure was applied for $10 \mathrm{~min}$, alternating $10 \mathrm{~s}$ negative pressure and $7 \mathrm{~s}$ atmospheric pressure ( 3 INP cycles per minute). Thereafter, a 5 -min post-INP sequence at atmospheric pressure was recorded.

All experiments were conducted in a quiet and temperature-controlled environment $\left(25 \pm 1.4{ }^{\circ} \mathrm{C}\right)$ to reduce sympathetic stress that could create artifacts [21] and to ensure thermoneutral conditions [22]. The participants' thermoneutral state was reflected by large fluctuations in the dorsal pedis artery blood flow velocity and high fluctuation frequencies in acral skin blood flow. Pressure within the subatmospheric pressure chamber was continuously monitored throughout the study using an attached calibrated pressure transducer (Fluke, 700G Series, Everett, WA, USA).

\section{Statistical analysis}

Descriptive data are presented as mean with (standard deviation) or median with (range), whichever suitable. We analyzed the data with a mixed-effects regression model to account for the repeated measurements within subjects, modeling the individual variations using random effects. Statistical analyses were performed using $\mathrm{R}$ version 3.3.3 with the "nlme" [23] and "multcomp" [24] add-on packages (R Foundation for Statistical Computing, Vienna, Austria). For all statistical tests, a two-tailed probability level of $P<$ 0.05 was considered statistically significant.

\section{Cross-validation of maximal blood flow}

One potential source of error when estimating maximal blood flow is that the estimated peak value can be inflated by random peaks when using the same data to both locate the maximum value and estimate its magnitude. To avoid this, we applied 8-fold cross-validation randomly, dividing the 24 subjects into 8 groups. We then systematically estimated the peak value for each (3-subject) group while locating the time to maximum flow and flux among the remaining 21 subjects. Flow and flux magnitude was then estimated by the average value across all the crossvalidation groups. To determine confidence bounds (intervals) for the estimated group differences, we sampled 10,000 bootstrapping replications from our patient material. Note that this was not performed for the estimates describing changes in flow over time within one INP cycle.

\section{Changes in flow over time within one negative pressure cycle}

To estimate the change in blood flow velocity and flux over time within one INP cycle, blood flow velocity and flux were bound within each second after the onset of negative pressure (second 0-17). Blood flow velocity and flux relative to the baseline sequence of each subject was evaluated in a mixed-effects model by assigning indicator variables to each second.

\section{Cumulative up-and-down in arterial blood flow velocity}

To evaluate the fluctuations (flow pulsatility) in arterial blood flow velocity $(\mathrm{cm} / \mathrm{s})$ during INP relative to baseline and post-INP, the differences were calculated between the average blood flow velocity during each heartbeat and the previous one. The sum of the absolute values of these differences for each subject were calculated within each sequence and averaged per minute, yielding the cumulative up-and-down variations in blood flow velocity per minute. By assigning variables to each sequence (baseline, INP, and post-INP), we compared the cumulative changes within each sequence in a mixed-effects model. Estimates and confidence intervals were calculated.

\section{Effects of the INP sequence averaged over the whole 10-min registration period}

For each subject, mean flow velocity $(\mathrm{cm} / \mathrm{s})$, laser Doppler flux (AU), skin temperature $\left({ }^{\circ} \mathrm{C}\right)$, HR (beats/min), and mean arterial pressure (MAP; $\mathrm{mm} \mathrm{Hg}$ ) were calculated for each sequence (baseline, INP, and post-INP, respectively). To remove disturbances in the transition zones, the first and last $10 \mathrm{~s}$ of each sequence were removed before analysis. Effects of the INP and post-INP sequences compared to baseline were evaluated in a mixed-effects regression model, by assigning variables to each sequence. 


\section{Results}

Adequate Doppler signals could not be obtained in 1 of the 25 recruited SCI participants. Twenty-four Caucasians were therefore included in the final arterial blood flow velocity analysis. Blood flow velocity was measured in the dorsal pedis artery $(n=23)$ and in the posterior tibial artery $(n=1)$. Participants' characteristics are presented in Table 1. The median (range) time with SCI was 19 years (range: 2.5-47) and the median (range) ABPI in the test leg was 1.0 (range: $0.42-1.10$ ). All participants were nondiabetic and non-smokers, and all were wheelchair users.

Table 1 Demographical characteristics $(n=24,12$ males and 12 females)

\begin{tabular}{lcl}
\hline Variables & Mean & $\begin{array}{l}\text { (Standard } \\
\text { deviation) }\end{array}$ \\
\hline Age (years) & 57 & $(12)$ \\
Body mass (kg) & 77 & $(16)$ \\
Height (cm) & 173 & $(12)$ \\
BMI (kg/m ${ }^{-2}$ ) & 26 & $(5.3)$ \\
Ankle-Brachial Index, test leg & 0.93 & $(0.1)$ \\
Ankle-Brachial Index, control leg & 0.92 & $(0.2)$ \\
PVR, test leg (mm) & 9.7 & $(4.0)$ \\
PVR, control leg (mm) & 10.6 & $(5.2)$ \\
Systolic blood pressure (mm Hg) & 118 & $(20)$ \\
Diastolic blood pressure (mm Hg) & 67 & $(8.9)$ \\
Mean arterial pressure (mm Hg) & 84 & $(11.0)$ \\
Time with spinal cord injury (years) & 21.6 & $(14)$ \\
SCI classification (complete/ & $13 / 11$ & \\
incomplete, $n)^{\mathrm{a}}$ & & \\
Level of injury (range) & & $(\mathrm{C} 4-\mathrm{Th} 12)$ \\
\hline
\end{tabular}

PVR pulse volume recording

${ }^{a}$ American Spinal Injury Association (ASIA score) [15]

\section{Cross-validation of maximal blood flow}

Estimated maximal blood flow velocity calculated by crossvalidation was $33 \%$ (95\% confidence interval (CI) 16 to 46, $P<0.001)$ above baseline. Estimated maximal skin blood flux (laser Doppler) was $11 \%$ (95\% CI -4.1 to 60 , $P=0.14)$ above baseline in the test leg, and $-0.49 \%$ (95\% CI -13 to $18, P=0.94)$ in the foot not exposed to INP.

\section{Blood flow velocity and laser Doppler flux changes during INP}

There were larger fluctuations in blood flow velocity during application of INP compared to baseline blood flow velocity (Fig. 2). Figure 3 presents changes in blood flow velocity and laser Doppler flux over time $(0-17 \mathrm{~s})$ during all INP cycles for all 24 participants (this gave a total of 847 analyzed INP cycles for all participants), relative to the baseline sequence for each participant. The flow velocity and flux values fluctuated above and below the mean baseline sequence values during INP (Figs. 2 and 3). Peak arterial blood flow velocity was reached $3 \mathrm{~s}$ after the onset of negative pressure, at $30 \%(95 \% \mathrm{CI} 22-38, P<0.001)$ above baseline (Fig. 3). At peak laser Doppler flux, this was $20 \%$ (95\% CI 5-36, $P<0.001$ ) above baseline (Fig. 3). Peak laser Doppler flux measured in the foot not exposed to INP was not significantly different from baseline, $P=0.17$ ) (Fig. 3).

\section{Cumulative up-and-down changes in arterial blood flow velocity}

Mean cumulative fluctuations in arterial blood flow velocity were $15.2 \mathrm{~cm} / \mathrm{s} / \mathrm{min}$ (95\% CI 9.8-20.6) in the baseline sequence. Compared to baseline, this increased to $32.3 \mathrm{~cm} /$ s/min $(95 \%$ CI $26.9-37.7, P<0.001)$ during INP. The postINP sequence flow velocity fluctuations were $18.3 \mathrm{~cm} / \mathrm{s} / \mathrm{min}$ (95\% CI 12.8-23.8, $P=0.17$, compared to baseline).
Fig. 2 The whole 20-min experiment for all participants $(n=24)$ with arterial blood flow velocities $(\mathrm{cm} / \mathrm{s})$ for each second relative to each participant's mean baseline value. Black lines are mean values with $95 \%$ confidence intervals as gray lines

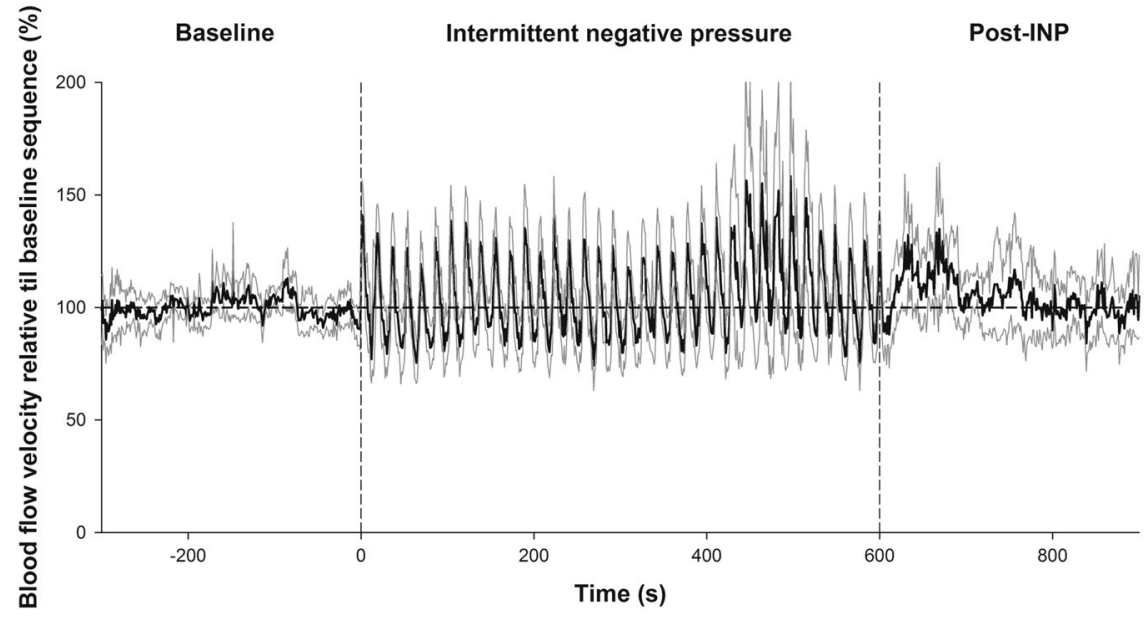


Fig. 3 The effects on blood flow velocity and laser Doppler flux in the test and the control leg, respectively, for the first $17 \mathrm{~s}$ (one pressure cycle) after onset of negative pressure $(-40 \mathrm{~mm}$ $\mathrm{Hg}$ ) among all SCI participants. One pressure cycle of INP equals $10 \mathrm{~s}$ of $-40 \mathrm{~mm} \mathrm{Hg}$ subatmospheric pressure and $7 \mathrm{~s}$ of atmospheric pressure. Note that time zero denotes first second after onset of negative pressure. The black dots represent mean values with $95 \%$ confidence intervals relative to the 5-min mean baseline sequence
Table 2 Physiological parameters for all the participants $(n=24)$ during the baseline, INP, and post-INP sequences
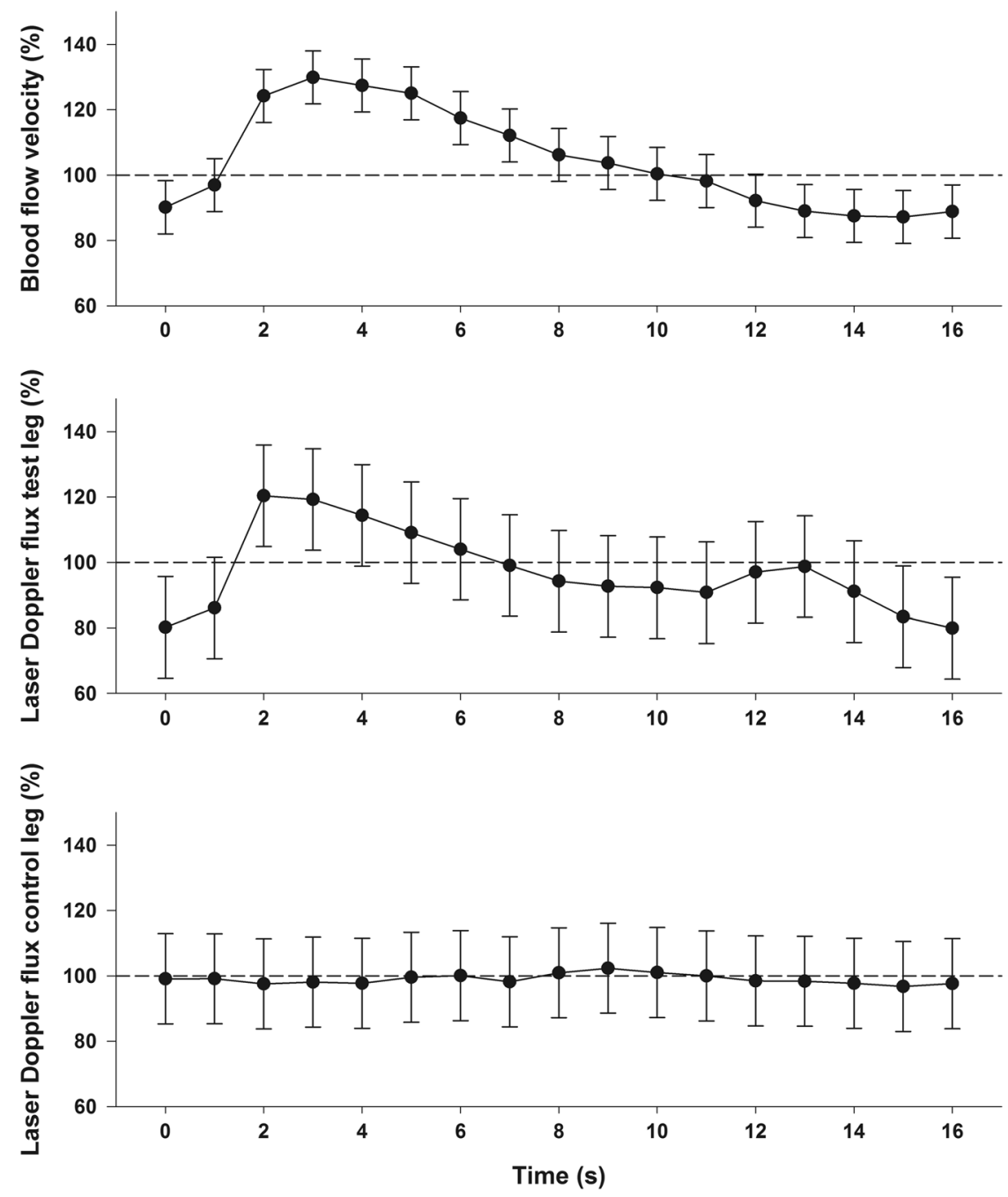

\begin{tabular}{|c|c|c|c|c|c|c|c|c|}
\hline \multirow[t]{2}{*}{ Parameters } & \multicolumn{2}{|l|}{ Baseline } & \multicolumn{3}{|l|}{ INP } & \multicolumn{3}{|l|}{ Post-INP } \\
\hline & Estimate & $95 \% \mathrm{CI}$ & Estimate & $95 \% \mathrm{CI}$ & $P$-value & Estimate & $95 \% \mathrm{CI}$ & $P$-value \\
\hline $\begin{array}{l}\text { Blood flow velocity } \\
(\mathrm{cm} / \mathrm{s})\end{array}$ & 5.2 & $3.8-6.3$ & 5.04 & $3.8-6.3$ & 0.93 & 5.22 & $4.0-6.0$ & 0.31 \\
\hline LDF test leg (AU) & 376 & $267-485$ & 329 & $219-438$ & 0.063 & 344 & $235-453$ & 0.2 \\
\hline LDF control leg (AU) & 527 & $340-714$ & 466 & $279-652$ & 0.05 & 507 & $320-694$ & 0.51 \\
\hline Skin temperature $\left({ }^{\circ} \mathrm{C}\right)$ & 32.4 & $31.4-33.4$ & 32.5 & $31.6-33.5$ & 0.025 & 32.8 & $31.8-33.7$ & $<0.001$ \\
\hline MAP (mm Hg) & 91 & $88-97$ & 91 & $84-97$ & 0.55 & 91 & $85-97$ & 0.79 \\
\hline HR (beats/min) & 67 & $62-72$ & 67 & $62-71$ & 0.16 & 66 & $61-70$ & 0.003 \\
\hline
\end{tabular}

Average estimates for the baseline, INP and post-INP sequences with $95 \%$ confidence intervals. $P$-values are used for comparison to the baseline sequence

$L D F$ laser Doppler flux, INP intermittent negative pressure, $A U$ arbitrary units, $M A P$ mean arterial pressure, $H R$ heart rate 


\section{Mean arterial blood flow}

Table 2 shows the estimates for the mean physiological values (arterial blood flow velocity, LDF, skin temperature, MAP, and HR) in different sequences (baseline, INP, and post-INP).

\section{Discussion}

The major finding of the present study was that INP applied to the lower leg and foot induced increased fluctuations (flow pulsatility) in arterial blood flow velocity in people with chronic SCI. INP induced an increase in peak arterial blood flow velocity at the onset of negative pressure, compared to average baseline blood flow velocity. Moreover, the increase in flow pulsatility occurred without significant changes in central hemodynamics. Our findings support the hypothesis that application of short oscillations of mild negative pressure to the lower leg and foot increases flow pulsatility in foot macrocirculation and microcirculation in people with SCI.

Application of INP to the lower limb has been reported to induce beneficial clinical changes in patients with peripheral vascular disease, such as wound healing, increased maximal walking distance, and resting flow after 4-6 weeks $[7,11]$. Those protocols involved INP treatment for half an hour two [7] or three times [11] per week. A double-blinded, randomized study [10] found increased ABPIs, maximal and pain-free walking distance, and beneficial effects on platelet aggregation in patients with peripheral arterial disease after 2 months with intermittent negative and positive pressure therapy to the lower body (Vacusac Intl. aps, Denmark). Similar findings were later replicated in peripheral arterial disease patients using the same method in a randomized, placebo-controlled crossover study [9]. Moreover, a recent case study on patients with late-stage peripheral arterial disease and chronic leg and foot ulcers (including one paraplegic patient) indicated that 8 weeks of INP therapy $2 \mathrm{~h}$ per day improves foot perfusion and wound healing [6]. These clinical findings are consistent with the INP-induced increase in foot circulation in two separate experimental studies: on healthy subjects [4] and on patients with peripheral arterial disease [5]. The present study demonstrates that the peripheral vasculature in SCI participants may have a comparable ability to increase macrocirculatory and microcirculatory foot circulation during INP as was shown for healthy subjects [4] and patients with atherosclerotic ischemic disease [5].

Previous investigations on healthy volunteers [4] and patients with peripheral arterial disease [5] found that INP induced an increase above baseline flow of $44 \%$ and $46 \%$, respectively. In the present study, INP increased blood flow velocity, with a cross-validated estimated peak level $27 \%$ above baseline.

The use of cross-validation, by picking randomly inflated points on the flow curve as the true peak, is important to avoid overestimating the true increase. Nevertheless, the approach might also underestimate peak flow, as crossvalidation might pick non-optimal peak locations in a relatively limited sample size. Hence, the true blood flow peak value is probably between our estimated value and the directly observed peak of $30 \%$ above the baseline level, at about $3 \mathrm{~s}$ after onset of negative pressure. After the peak, flow velocity gradually declined, despite continued negative pressure. After about $11 \mathrm{~s}$, blood flow velocity fell below baseline values. This decline in blood flow velocity below baseline values reduced mean arterial blood flow velocity during the INP sequence.

We have previously found that INP applied through an identical 10-min INP sequence as in the present study induced a $12 \%$ increase in flow velocity in patients with peripheral arterial disease, compared to 5-min baseline flow [5]. The explanation for this difference is not clear, but it is possible that there is a stronger local vasoconstrictor response to venous distension in the lower limbs among people with SCI than among able-bodied individuals [14].

The mechanisms of action for the observed increased flow pulsatility during INP in the present study are not clear. However, the proposed main contributing mechanisms to the increase in flow during suction are an increase in perfusion pressure $[7,8]$ and avoidance of the local venoarteriolar reflex [25], which constricts the arterioles when the veins are distended during constant negative pressure [26]. This vasoconstrictor response is independent of brain stem control and is also found intact in subjects with tetraplegia [27].

As previously shown, pressure changes within a subatmospheric pressure chamber are accompanied by similar pressure changes in the superficial veins and interstitial tissues within the limb exposed to subatmospheric pressure [7, 8]. According to Poiseuille's law [28], blood flow may be increased by changing perfusion pressure or vascular resistance or through a combination of these two factors. Increased flow pulsatility during INP is therefore likely explained-at least partially-by temporary changes in perfusion pressure within the vessels during application of subatmospheric pressure [7].

By applying INP to the lower leg and foot, increased arterial inflow during suction is likely to occur in all six angiosomes and collateral vessels of the lower leg and foot. This is due to rapid changes in perfusion pressure and mechanical dilation of the tissues and vessels exposed to INP. The abrupt INP-induced change in ambient pressure induces increased flow pulsatility, which provides variable driving pressure for blood flow through the cutaneous tissue $[29,30]$. Blood flow through the cutaneous tissue, in turn, 
results in short bursts of arterial blood flow to the small vessels of the skin. Flow pulsatility is found to: (i) improve erythrocyte flow velocity in the capillaries; (ii) increase the number of perfused capillaries; (iii) increase tissue oxygenation; (iv) reduce peripheral vascular resistance; and (v) enhance basal and flow-stimulated endothelium-derived nitric oxide release in the peripheral vasculature in vivo [31]. Such fluctuations in blood flow have been proposed to be more essential than mean flow for adequate tissue perfusion [32, 33].

In recent years, the use of focal negative pressure wound therapy (NPWT) has become a common worldwide modality in the treatment of acute and chronic wounds [34], despite scarce evidence of its effects on leg ulcer healing [34]. NPWT applies subatmospheric pressure to the local wound environment through a sealed dressing and sucks wound and tissue fluid into a canister [34]. NPWT is different from the INP method investigated in the present paper for three main reasons: First, NPWT only applies subatmospheric pressure to a focal wound area. Second, NPWT commonly applies higher subatmospheric pressures ( -80 to $-125 \mathrm{~mm} \mathrm{Hg}$ ) [35] than used in the present study. Third, NPWT applies subatmospheric pressures continously or with a cycle length of minimum $\geq 2$ min.

We have previously demonstrated that application of 2min constant negative pressure decreases arterial and skin blood flow in the foot of healthy subjects, while short oscillations of $10 \mathrm{~s}$ negative pressure increased arterial and skin blood flow in the foot [4]. Further, Skagen et al. [26] found that constant negative pressure applied to the skin resulted in increased vascular resistance and a pronounced decrease in skin blood flow. Accordingly, NPWT decreases tissue perfusion beneath the wound dressing [36] and in close proximity to the wound area [37] and decreases tissue oxygenation of the foot $[38,39]$.

\section{Methodological considerations}

There are some limitations to the present study. First, arterial blood flow velocities were used to describe changes in arterial blood flow in the foot in the participants. We were not able to measure the diameter of the dorsalis pedis and posterior tibial arteries within the subatmospheric pressure chamber during INP. However, pulsatile diameter in small arteries has been found to be very stable [40]. We therefore believe it is reasonable to assume that the changes in observed blood flow velocity during INP reflect changes in blood flow in the foot artery. If vessel diameter were to increase due to flow-mediated vasodilation, this would tend to further increase arterial blood flow during INP and therefore underestimate our INP-induced flow effect. We observed in the present study increased flow pulsatility in both blood flow velocity and laser Doppler flux of the foot during the INP cycles compared to baseline. Together, these findings demonstrate that INP induced skin perfusion of the foot during INP.

Second, we recruited mainly from the local hospital training group. The SCI population in the present study consisted of non-smokers and non-diabetics without leg or foot ulcers. The recruited population in our study may therefore have been more physically fit and active than the average SCI person. Many people with SCI have abnormal carbohydrate and lipid metabolism and higher rates of cardiovascular disease [41], due to deconditioning and extreme inactivity, as well as possibly due to higher smoking rates compared to the general population [42].

\section{Conclusions}

This study found that application of mild INP to the lower leg and foot induced a significant, abrupt increase in arterial blood flow in the foot, followed by a decrease. This fluctuation was observed following the onset of negative pressure, without any significant changes in MAP or HR. This INP-induced increase in blood flow pulsatility during short oscillations of negative pressure may potentially promote tissue perfusion and ulcer healing in SCI people with ulcers in the lower limb. Future studies are warranted to explore possible clinically relevant effects and to elicit the working mechanisms of INP.

Acknowledgements The authors would like to thank the patients for their participation in this study. We appreciate Otivio AS for procuring laboratory probes, subatmospheric pressure chambers, and INP pumps for use in the study. We would also like to thank Øystein Horgmo at the Medical Photography Section at the Institute of Clinical Medicine, University of Oslo for help with the illustrations and Annie Bersagel for language editing.

Author contributions $\varnothing \mathrm{HS}, \mathrm{L} \varnothing \mathrm{H}, \mathrm{JH}$, and HW-F conceived of and designed the study. ØHS performed the experiments. ØHS, LØ, JH, HW-F, II, and HH, analyzed the data; ØHS, LØH, JH, and II interpreted the results of the experiments; $\mathrm{L} \varnothing \mathrm{H}, \varnothing \mathrm{HS}$, and JH prepared the figures; $\varnothing \mathrm{HS}$ wrote the manuscript. All authors edited and critical reviewed the manuscript and approved the final version of the manuscript.

Funding The Research Council of Norway provided funding to Otivio (NFR grant no: 241589) for this study as part of an industrial PhD project at the Univerity of Oslo.

\section{Compliance with ethical standards}

Conflict of interest $\varnothing \mathrm{HS}$ is employed at and is a shareholder in Otivio AS. Otivio AS owns and has the commercial rights to the intermittent negative pressure (INP) technology used in the study. IM is the CSO and co-founder of Otivio AS and is a shareholder in the company. None of the other authors have any personal conflicts of interestfinancial or otherwise. The authors alone are responsible for the content and writing of the paper. Otivio has not had any role related to the 
design of the study, collection and analysis of data, or the decision to publish the results.

Ethical approval The Regional Committee for Medical and Health Research Ethics in Norway approved the experimental protocols (protocol number: 2014/1967). The study was performed in accordance with the Declaration of Helsinki.

Informed consent Written and oral informed consent was obtained from all participants.

\section{References}

1. Basson MD, Burney RE. Defective wound healing in patients with paraplegia and quadriplegia. Surg Gynecol Obstet. 1982;155:9-12.

2. Rappl LM. Physiological changes in tissues denervated by spinal cord injury tissues and possible effects on wound healing. Int Wound J. 2008;5:435-44.

3. Deitrick G, Charalel J, Bauman W, Tuckman J. Reduced arterial circulation to the legs in spinal cord injury as a cause of skin breakdown lesions. Angiology. 2007;58:175-84.

4. Sundby $\varnothing \mathrm{H}$, Høiseth LØ, Mathiesen I, Jørgensen JJ, WeedonFekjær H, Hisdal J. Application of intermittent negative pressure on the lower extremity and its effect on macro- and microcirculation in the foot of healthy volunteers. Physiol Rep. 2016;4: e12911.

5. Sundby $\varnothing H$, Høiseth Lø, Mathiesen I, Weedon-Fekjær H, Sundhagen JO, Hisdal J. The acute effects of lower limb intermittent negative pressure on foot macro- and microcirculation in patients with peripheral arterial disease. PLoS ONE. 2017;12: $\mathrm{e} 0179001$.

6. Sundby $\varnothing \mathrm{H}$, Høiseth LØ, Mathiesen I, Jørgensen JJ, Sundhagen $\mathrm{JO}$, Hisdal J. The effects of intermittent negative pressure on the lower extremities' peripheral circulation and wound healing in four patients with lower limb ischemia and hard-to-heal leg ulcers: a case report. Physiol Rep. 2016;4:e12998.

7. Smyth CN. Effect of suction on blood-flow in ischaemic limbs. Lancet. 1969;2:657-9.

8. Caro CG, Foley TH, Sudlow MF. Early effects of abrupt reduction of local pressure on the forearm and its circulation. J Physiol. 1968;194:645-58.

9. Himmelstrup H, Himmelstrup B, Mehlsen J, Trap-Jensen J. Effects of vacusac in intermittent claudication: a controlled crossover study. Clin Physiol. 1991;11:263-9.

10. Mehlsen J, Himmelstrup H, Himmelstrup B, Winther K, TrapJensen J. Beneficial effects of intermittent suction and pressure treatment in intermittent claudication. Angiology. 1993;44:16-20.

11. Gill BS, Walder DN. Proceedings: the effect of intermittent suction on limb blood flow in peripheral vascular disease. Br J Surg. 1974;61:319.

12. Partida E, Mironets E, Hou S, Tom VJ. Cardiovascular dysfunction following spinal cord injury. Neural Regen Res. 2016;11:189-94.

13. Olive JL, McCully KK, Dudley GA. Blood flow response in individuals with incomplete spinal cord injuries. Spinal Cord. 2002;40:639-45.

14. Kooijman M, de Hoog M, Rongen GA, van Kuppevelt HJ, Smits P, Hopman MT. Local vasoconstriction in spinal cord-injured and able-bodied individuals. J Appl Physiol (1985). 2007;103:1070-7.

15. Maynard FM Jr., Bracken MB, Creasey G, Ditunno JF Jr., Donovan WH, Ducker TB, et al. International Standards for Neurological and Functional Classification of Spinal Cord Injury.
American Spinal Injury Association. Spinal Cord. 1997;35:266-74.

16. Fagius J, Karhuvaara S. Sympathetic activity and blood pressure increases with bladder distension in humans. Hypertension. 1989;14:511-7.

17. Aboyans V, Criqui MH, Abraham P, Allison MA, Creager MA, Diehm $\mathrm{C}$, et al. Measurement and interpretation of the anklebrachial index: a scientific statement from the American Heart Association. Circulation. 2012;126:2890-909.

18. Hirsch AT, Haskal ZJ, Hertzer NR, Bakal CW, Creager MA, Halperin JL, et al. ACC/AHA 2005 Practice Guidelines for the management of patients with peripheral arterial disease (lower extremity, renal, mesenteric, and abdominal aortic): a collaborative report from the American Association for Vascular Surgery/ Society for Vascular Surgery, Society for Cardiovascular Angiography and Interventions, Society for Vascular Medicine and Biology, Society of Interventional Radiology, and the ACC/AHA Task Force on Practice Guidelines (Writing Committee to Develop Guidelines for the Management of Patients With Peripheral Arterial Disease): endorsed by the American Association of Cardiovascular and Pulmonary Rehabilitation; National Heart, Lung, and Blood Institute; Society for Vascular Nursing; TransAtlantic Inter-Society Consensus; and Vascular Disease Foundation. Circulation. 2006;113:e463-654.

19. Hafner J, Schaad I, Schneider E, Seifert B, Burg G, Cassina PC. Leg ulcers in peripheral arterial disease (arterial leg ulcers): impaired wound healing above the threshold of chronic critical limb ischemia. J Am Acad Dermatol. 2000;43:1001-8.

20. Sarnik S, Hofirek I, Sochor O. Laser Doppler fluxmetry. Biomed Pap Med Fac Univ Palacky Olomouc Czech Repub. 2007;151:143-6.

21. Thoresen M, Walloe L. Skin blood flow in humans as a function of environmental temperature measured by ultrasound. Acta Physiol Scand. 1980;109:333-41.

22. Elstad M, Vanggaard L, Lossius AH, Walloe L, Bergersen TK. Responses in acral and non-acral skin vasomotion and temperature during lowering of ambient temperature. $\mathrm{J}$ Therm Biol. 2014;45:168-74.

23. Pinheiro J, Bates D, DebRoy S, Sarkar D, Team RC. nlme: Linear and nonlinear mixed effects models. R package version 3.1-128. Vienna: R Foundation for Statistical Computing, 2016. .

24. Hothorn T, Bretz F, Westfall P. Simultaneous inference in general parametric models. Biom J. 2008;50:346-63.

25. Rein EB, Filtvedt M, Walloe L, Raeder JC. Hypothermia during laparotomy can be prevented by locally applied warm water and pulsating negative pressure. $\mathrm{Br} \mathrm{J}$ Anaesth. 2007;98:331-6.

26. Skagen K, Henriksen O. Changes in subcutaneous blood flow during locally applied negative pressure to the skin. Acta Physiol Scand. 1983;117:411-4.

27. Skagen K, Jensen K, Henriksen O, Knudsen L. Sympathetic reflex control of subcutaneous blood flow in tetraplegic man during postural changes. Clin Sci (Lond). 1982;62:605-9.

28. Pfitzner J. Poiseuille and his law. Anaesthesia. 1976;31:273-5.

29. Undar A, Masai T, Frazier OH, Fraser CD Jr. Pulsatile and nonpulsatile flows can be quantified in terms of energy equivalent pressure during cardiopulmonary bypass for direct comparisons. ASAIO J. 1999;45:610-4.

30. Barić D. Why pulsatility still matters: a review of current knowledge. Croat Med J. 2014;55:609-20.

31. Nakano T, Tominaga R, Nagano I, Okabe H, Yasui H. Pulsatile flow enhances endothelium-derived nitric oxide release in the peripheral vasculature. Am J Physiol Heart Circ Physiol. 2000;278:H1098-104. 
32. Stefanovska A. Dynamics of blood oxygenation gives better insight into tissue hypoxia than averaged values. Am J Physiol Heart Circ Physiol. 2009;296:H1224-6.

33. Tsai AG, Intaglietta M. Evidence of flowmotion induced changes in local tissue oxygenation. Int $\mathrm{J}$ Microcirc Clin Exp. 1993;12:75-88.

34. Dumville JC, Land L, Evans D, Peinemann F. Negative pressure wound therapy for treating leg ulcers. Cochrane Database Syst Rev. 2015;(7):CD011354.

35. Vikatmaa P, Juutilainen V, Kuukasjarvi P, Malmivaara A. Negative pressure wound therapy: a systematic review on effectiveness and safety. Eur J Vasc Endovasc Surg. 2008;36: 438-48.

36. Kairinos N, Voogd AM, Botha PH, Kotze T, Kahn D, Hudson DA, et al. Negative-pressure wound therapy II: negative-pressure wound therapy and increased perfusion. Just an illusion? Plast Reconstr Surg. 2009;123:601-12.

37. Borgquist O, Ingemansson R, Malmsjo M. Wound edge microvascular blood flow during negative-pressure wound therapy: examining the effects of pressures from -10 to $-175 \mathrm{mmHg}$. Plast Reconstr Surg. 2010;125:502-9.

38. Shon YS, Lee YN, Jeong SH, Dhong ES, Han SK. Influence of negative-pressure wound therapy on tissue oxygenation of the foot. Arch Plast Surg. 2014;41:668-72.

39. Jung JA, Yoo KH, Han SK, Lee YN, Jeong SH, Dhong ES, et al. Influence of negative-pressure wound therapy on tissue oxygenation in diabetic feet. Adv Skin Wound Care. 2016;29:364-70.

40. Eriksen M. Effect of pulsatile arterial diameter variations on blood flow estimated by Doppler ultrasound. Med Biol Eng Comput. 1992;30:46-50.

41. Cragg JJ, Noonan VK, Krassioukov A, Borisoff J. Cardiovascular disease and spinal cord injury: results from a national population health survey. Neurology. 2013;81:723-8.

42. Saunders LL, Krause JS, Saladin M, Carpenter MJ. Prevalence of cigarette smoking and attempts to quit in a population-based cohort with spinal cord injury. Spinal Cord. 2015;53:641-5. 